\title{
Novas funcionalidades do Sistema de Modelagem Física Multicanal da UENF/LENEP
}

Andrade, S. D.R. ${ }^{1,2}$, Missagia, R. M. ${ }^{1}$, Ceia, M. A. R. ${ }^{1}$,

${ }^{1}$ Universidade Estadual do Norte Fluminense - Darcy Ribeiro, ${ }^{2}$ Instituto Federal Fluminense - IFF/Macaé/RJ

Copyright 2018, SBGf - Sociedade Brasileira de Geofísica

Este texto foi preparado para a apresentação no VIII Simpósio Brasileiro de Geofísica, Salinópolis, 18 a 20 de setembro de 2018. Seu conteúdo foi revisado pelo Comitê Técnico do VIII SimBGf, mas não necessariamente representa a opinião da SBGf ou de seus associados. É proibida a reprodução total ou parcial deste material para propósitos comerciais sem prévia autorização da SBGf.

\section{Resumo}

Este trabalho apresenta um projeto para montagem de um protótipo e também o resultado da conversão de um Sistema de Modelagem Física de Mono para Multicanal, ambos usados para aquisição de dados sísmicos de reflexão em laboratório. Desta forma, estão sendo apresentados a metodologia empregada e a análise comparativa entre os resultados obtidos com ensaios antes e depois da conversão do Sistema. Os resultados revelam o impacto positivo a partir da obtenção de dados sísmicos ultrassônicos de alta resolução que pode ser atribuído a incorporação de novas funcionalidades (hardwere e softwere) ao sistema de modelagem.

\section{Introdução}

A sísmica de reflexão tem sido empregada em larga escala na exploração e produção de hidrocarbonetos para obtenção de informações acerca das rochas reservatórios, auxiliando a redução de incertezas associadas a problemas relacionados ao mapeamento de estruturas sísmicas (locação de poços produtores e injetores), e caracterização de reservatórios (monitoramento de reservatórios de óleo e gás), (Assis et al., (2015)).

De acordo com Misságia et al., (2010), os experimentos de modelagem física ultrassônica têm sido usados como ferramenta efetiva para melhorar o entendimento acerca dos fenômenos físicos que ocorrem no campo, durante etapas da exploração e produção de reservatórios portadores de óleo e gás. Isto é possível a partir da análise da expressão sísmica ultrassônica, advinda da simulação da propagação da onda em modelos físicos construídos por materiais com propriedades análogas a rocha natural sob investigação. Tais experimentos propiciam confiáveis correlações entre dados de campo e de laboratório, com potencial para viabilizar a redução de custos e mitigar incertezas.

Desta forma é importante controlar todas as variáveis envolvidas na aquisição de dados sísmicos, para garantir a confiabilidade e contribuir para a interpretação dos eventos presentes nas seções sísmicas.

Segundo Cordsen et al, (2000) a precisão no posicionamento dos dados sísmicos, possibilita maior repetibilidade e incremento na razão sinal/ruído.

Existem muitos trabalhos que utilizam este tipo de modelagem com o objetivo de testar algoritmos de processamento sísmico, melhorar técnicas de aquisição e observar a resposta sísmica do modelo.

Cooper et al., (2010) e Missagia et al., (2011) investigaram modelos físicos na forma de cunha, para verificar como a amplitude dos eventos de reflexão varia quando a espessura da camada diminui para valores abaixo do comprimento de onda dominante. Assis, (2015) calibrou o transdutor semi-esférico para aquisição de dados de reflexão voltados para a análise AVO, fornecendo uma forma de onda aproximadamente constante para um grande intervalo de ângulos de emissão/registro, além do baixo padrão de directividade. Nesta dissertação a aquisição e processamento dos dados ultrassônicos foram idealizados para remover os eventos lineares que interferem nas reflexões alvo, compensar perdas de amplitudes causadas pelo efeito da directividade fonte/receptor, espalhamento geométrico e perdas de transmissão. Zambrinni (2015) deu ênfase a migração e análise do atributo de atenuação sísmica em modelo físico. Silva (2013). Modelou os efeitos da saturação heterogênea para análise da sensibilidade da resposta sísmica ultrassônica devido ao efeito da variação granulométrica dos grãos que compõe o arcabouço do modelo físico e o impacto no sistema poroso.

\section{O Sistema de Modelagem Física}

A modelagem física sísmica tem sido empregada em universidades há mais de 80 anos (Misságia et al., 2010).

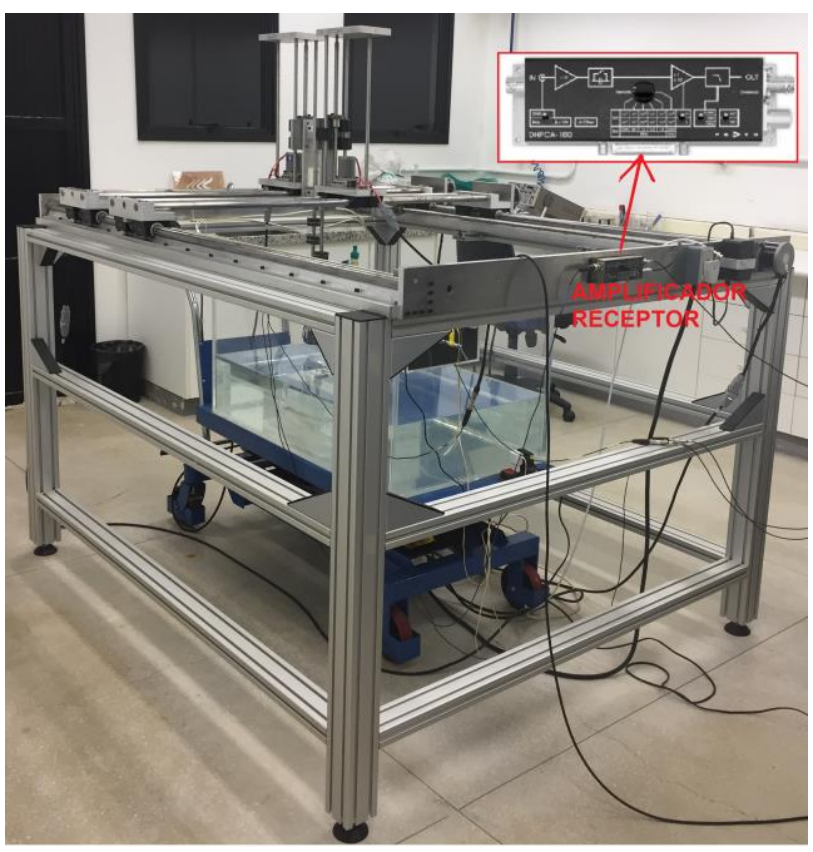

Figura 1- Mesa de Coordenadas $X Y Z$ 
$\mathrm{Na}$ Universidade Estadual do Norte Fluminense - UENF, em Macaé - RJ, experimentos sísmicos têm sido realizados em laboratório por um sistema que possui uma mesa de coordenadas $\mathrm{X}, \mathrm{Y}$ e $\mathrm{Z}$ em aço, com um tanque de água, inserido no meio desta estrutura (Figura 1).

As pesquisas desenvolvidas no LENEP/UENF com a utilização do sistema de Modelagem Física Monocanal foram determinantes para evidenciar 0 potencial aquisição e processamento do dado sísmico de laboratório, indicando o aprimoramento e conversão do Sistema de Modelagem Sísmica de Mono para Multicanal. Deste modo, neste trabalho está sendo apresentado todas as modificações realizadas no hardware e software que permitiram a conversão do sistema.

\section{Metodologia}

Visando aquisições sísmicas 3D com mais rapidez, precisão no posicionamento dos sensores e dados com aumento na relação sinal/ruído, a Figura 2 mostra o diagrama do protótipo para montagem integral de um Sistema de Modelagem Física Multicanal, incluindo as especificações de hardware e de software (Figura 5). Conforme relatado na seção anterior, neste trabalho somente a adaptações e mudanças no atual sistema de modelagem monocanal foram implementadas até 0 presente momento.

\section{Hardware}

O sistema monocanal possui uma mesa de coordenadas XYZ (Figura 1) e um conjunto de componentes e equipamentos mostrados na Figura 3, para gerenciar e controlar a aquisição de dados e movimentação dos transdutores ultrassônicos durante um levantamento sísmico no laboratório.

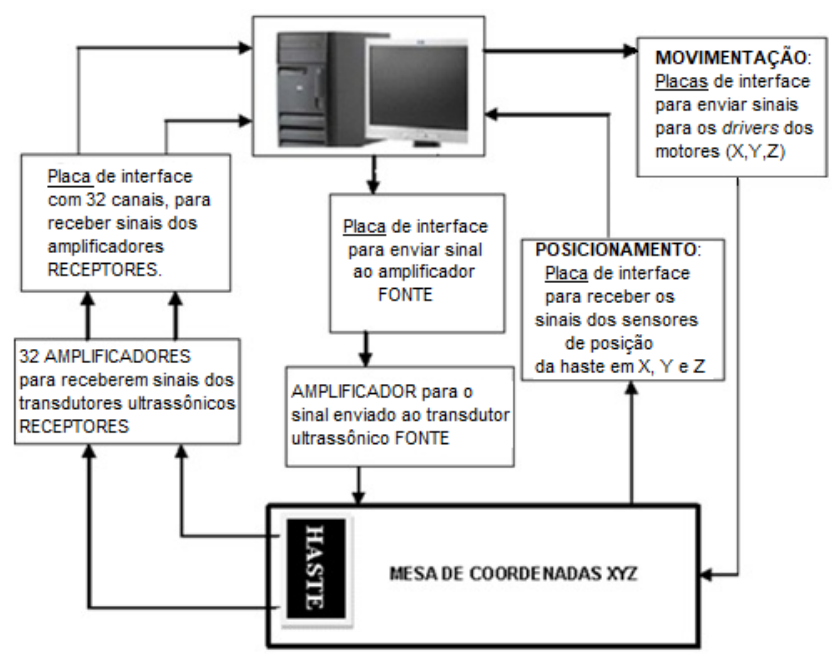

Figura 2 - Diagrama total das partes físicas do sistema de modelagem multicanal.

A metodologia adotada para o funcionamento do sistema como multicanal foi substituir a placa de interface digitalanalógica (DA) usada para a recepção do sinal ultrassônico.

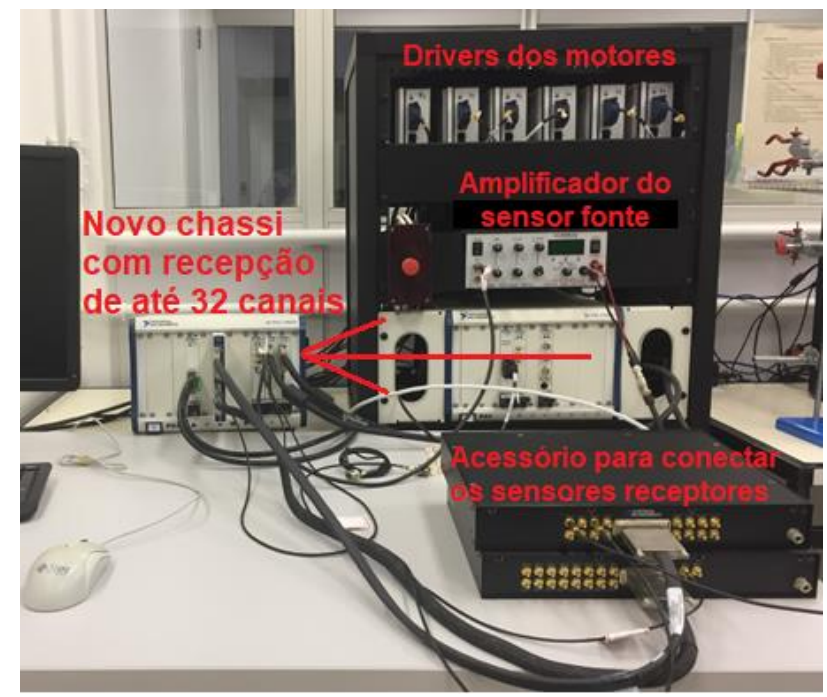

Figura 3 - Chassi com placas eletrônicas de interface entre o computador e a mesa de coordenadas $X Y Z$.

Com esta alteração, 0 atual chassi teve que ser substituído, pois o barramento interno para tráfego de dados não suporta a quantidade de dados sísmicos recebidos por 32 canais.

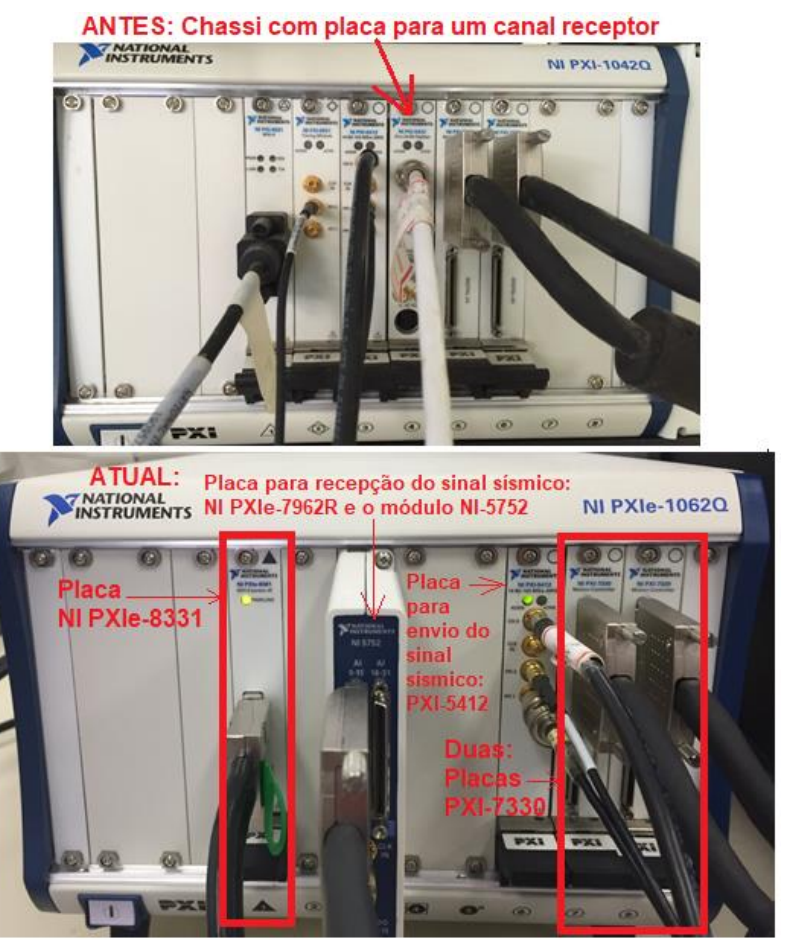

Figura 4 - Chassi com placa monocanal, na parte superior, e chassi atual com todas as placas de interface para o sistema de modelagem multicanal na parte inferior.

Por isso, foi adquirido o novo chassi NI PXle-1062Q e na Figura 3, a seta vermelha aponta para o novo gabinete que comporta as placas de interface. Na Figura 4 é mostrado o gabinete do sistema monocanal, que foi 
substituído pelo atual gabinete que possui a placa com até 32 canais de recepção.

Para receber os 32 canais de forma simultânea é necessário acoplar duas placas em um mesmo compartimento (slot), dentro do novo chassi: placas NI5752 e NI PXle-7962R. A placa NI PXle-7962R possui o chip FPGA (Field Programmable Gate Array) que oferece E/S (entradas e saídas) customizáveis e flexíveis, permitindo o usuário configurar o chip FPGA, conforme sua aplicabilidade.

Além disso, o uso da tecnologia FPGA possibilita a transferência de dados diretamente com o módulo $\mathrm{NI}$-5752, sem enviar dados para o processador do computador, agilizando o processamento dos dados recebidos dos 32 canais simultaneamente.

As novas placas de interface receptoras multicanais: NI5752 e NI PXle-7962R, foram instaladas no novo chassi (Figura 4) junto as partes físicas do sistema monocanal (Figura 1 e 3) para compor o atual sistema de modelagem física sísmica multicanal. Também foram adquiridos dois acessórios, mostrados na Figura 3, os quais recebem diretamente os transdutores ultrassônicos conectados por cabos que adaptam os conectores SMB para BNC. Cada um dos acessórios pode conectar até 16 transdutores.

Nesta etapa de adequação, além da inserção das novas placas de recepção também foi necessário retirar três placas do chassi anterior e transferir para o novo chassi, viabilizando que o sistema multicanal funcione em apenas um chassi (Figura 4).

As placas que foram transferidas são para movimentação dos motores: duas NI PXI-7330 e uma para o envio do sinal ao transdutor fonte: NI PXI-5412. As partes físicas que foram mantidas do sistema de modelagem monocanal são: os drivers dos motores, a mesa de coordenadas $\mathrm{XYZ}$, o amplificador para enviar o sinal sísmico ao transdutor fonte e o amplificador para um único canal de recepção (mostrado na Figura 1) das ondas sísmicas refletidas, captadas pelo transdutor ultrassônico.

\section{Software}

No computador o software desenvolvido na plataforma $\angle A B V I E W$ permite a integração entre as placas eletrônicas de interface e as partes físicas para gerenciar a movimentação dos motores e, os sinais enviados e recebidos dos sensores ultrassônicos, conforme mostra o fluxograma da Figura 5.

A Figura 6 exibe as telas gráficas de controle do sistema de modelagem pelo computador. O software monitora e gerencia o acesso entre o processo de movimentação da haste com os transdutores e o de aquisição de dados para executar um experimento sísmico. O movimento dos transdutores acontece de acordo com a parametrização da geometria de aquisição inserida no programa pelo usuário do sistema de modelagem.

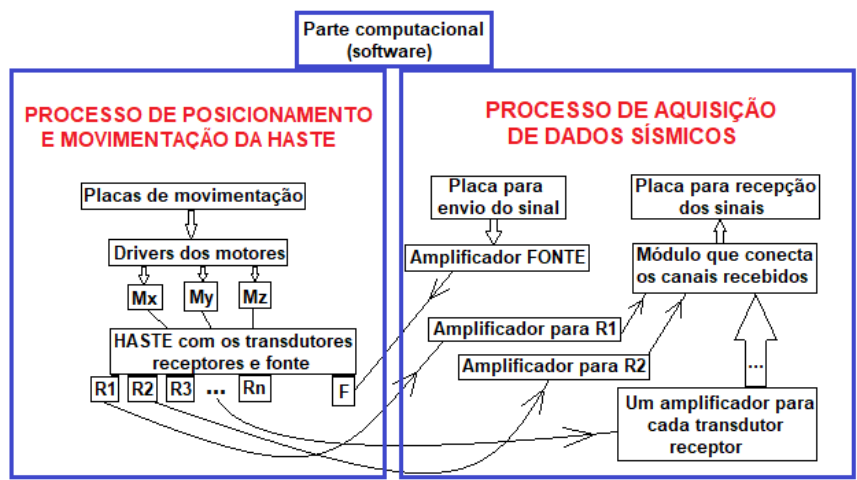

Figura 5 - Esquema do fluxo de funcionamento computacional, do sistema multicanal, para realizar um levantamento sísmico.

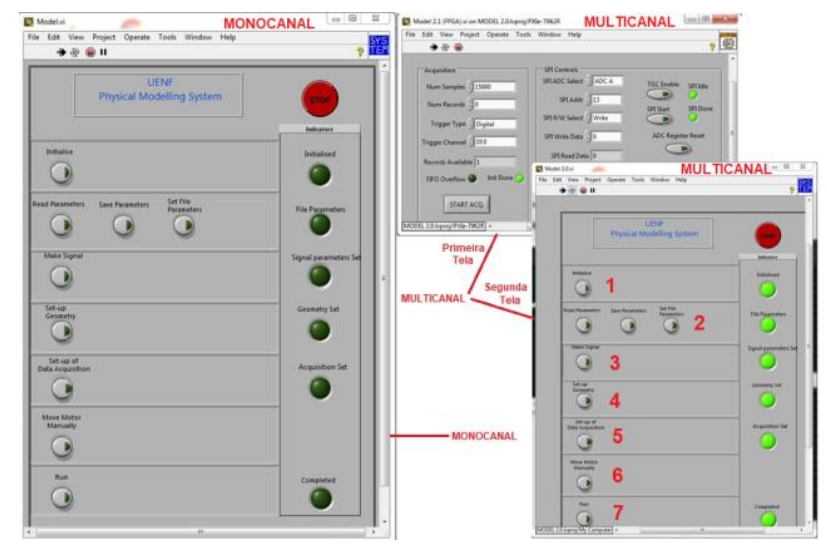

Figura 6 - Telas gráficas de integração com o usuário.

A tela principal, que está numerada à direita da Figura 6 , possui botões que acessam outras telas usadas na definição dos parâmetros de execução de um experimento sísmico. A Figura 6 mostra que no sistema monocanal apenas uma tela principal era aberta para a execução do software de aquisição. No sistema multicanal, duas telas são necessárias para parametrizar todo o experimento. Uma tela gráfica é usada apenas para levar a programação no chip FPGA para a placa de recepção dos 32 canais conectados aos transdutores. A outra tela é a principal, que contém sete etapas para o sistema de modelagem física sísmica funcionar movimentando os motores, enviando o sinal sísmico ao transdutor fonte e recebendo os sinais sísmicos refletidos, captados pelos transdutores ultrassônicos. De igual forma, estas etapas também eram processadas no sistema monocanal. A diferença entre os dois sistemas, está na etapa 5 (Figura 6) de recepção dos canais.

Durante as modificações realizadas no software para a leitura e gravação dos dados recebido nos canais da placa de interface, foi verificado que durante o registro do sinal, se os motores ficassem ligados, muito ruído era inserido no sinal recebido. Por isso, a rotina de execução do software foi alterada. No atual sistema multicanal, quando for realizado um levantamento sísmico, os motores serão totalmente desligados durante o envio e recepção dos sinais dos sensores ultrassônicos. Esta modificação diminuiu o tempo de execução do 
levantamento sísmico pois com 12 tiros em uma mesma posição, um traço sísmico é gerado por empilhamento desta família de traços, aumentando a relação sinal/ruído. Com os motores ligados durante todo o tempo do experimento, o sistema monocanal, necessitava de aplicar 240 ou mais tiros, em uma mesma posição, para melhorar o sinal recebido. O atual sistema multicanal está usando três dos 32 canais da placa de interface receptora, pois no laboratório existem apenas quatro sensores ultrassônicos com características iguais (Sensor da Panasonic V103 de face plana). Um está sendo usado como emissor e três como receptores (Panametrics, 2009). Mas a capacidade deste sistema de modelagem multicanal pode ser expandida para até 96 canais receptores, a partir da compra de mais duas placas de interface de recepção do sinal ultrassônico. Assim, o atual chassi NI PXle-1062Q poderá receber sinais de até 96 transdutores (Figuras 3, 4 e 5).

\section{Resultados}

Após a implementação proposta no hardware e software para conversão do Sistema de Modelagem Física Sísmica Mono para Multicanal, os dados sísmicos ultrassônicos de Silva, (2013) foram novamente adquiridos para viabilizar a análise comparativa e evidenciar as características e melhorias advindas desta conversão. Nestes experimentos sísmicos foi adotada a escala de 1:10.000.

Durante os testes para calibrar as novas componentes do sistema de modelagem, foi observado no osciloscópio que o sinal transmitido poderia se aproximar mais de um sinal analógico (contínuo). A Figura 7 possibilita a verificação da diferença entre o uso das diferentes taxas de amostragem. Assim, o dado do sinal transmitido foi alterado no atual sistema multicanal, pois a placa de envio: NI PXI-5412 possui uma taxa de amostragem de até $100 \mathrm{M}$ Samples/seg. Na Tabela 1 é possível ver esta mudança na parametrização: de $4 \mathrm{MS} / \mathrm{s}$ para $40 \mathrm{MS} / \mathrm{s}$ e a quantidade de amostras de 5.000 para 50.000 , dados que não afetaram no tempo de envio do sinal, mantido em $1,25 \mathrm{~ms}$.

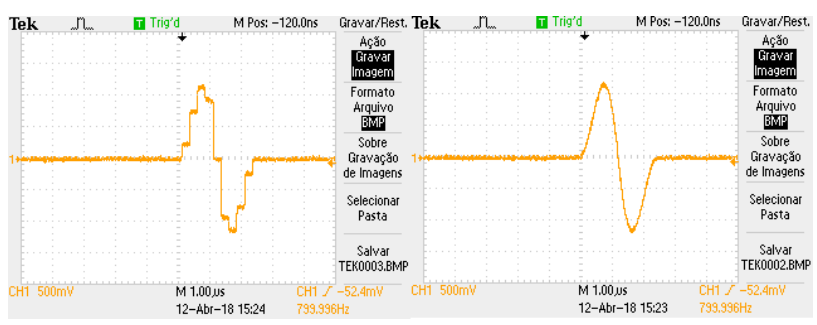

Figura 7 - Sinal transmitido Tone Burst, com a Taxa de $4 \mathrm{MS} / \mathrm{s}$ à esquerda e $40 \mathrm{MS} / \mathrm{s}$ à direita.

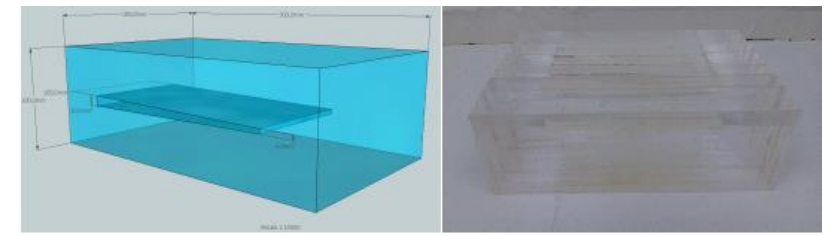

Figura 8 - À esquerda, o desenho do modelo físico geológico com um reservatório tipo cunha (pinch-out).

No LENEP/UENF foi desenvolvida uma pesquisa com a modelagem física sísmica, para analisar a sensibilidade da resposta sísmica devido ao efeito que a variação granulométrica provoca no meio poroso (Silva, 2013). A Figura 8, ilustra um modelo físico geológico com um reservatório tipo cunha (pinch-out), que foi usado neste estudo inicialmente, sem usar as esferas de vidro (meio poroso) para identificar eventos no sismograma.

Tabela 1 - Parâmetros usados na aquisição de dados.

\begin{tabular}{|c|c|c|c|}
\hline \multirow{2}{*}{ Parâmetros } & \multicolumn{2}{|c|}{ Dados em laboratório } & \multirow{2}{*}{$\begin{array}{c}\text { Dados } \\
\text { de } \\
\text { Campo }\end{array}$} \\
\hline & Monocanal & Multicanal & \\
\hline Tipo de sinal transmitido & Tone Burst & Tone Burst & \\
\hline 힌 Frequência de amostragem & $4 \mathrm{MS} / \mathrm{seg}$ & $40 \mathrm{MS} / \mathrm{seg}$ & \\
\hline Quantidade de amostras & 5.000 & 50.000 & \\
\hline \begin{tabular}{l|l} 
& \\
$\infty$ & Tempo de envio do sinal \\
¿
\end{tabular} & $1,25 \mathrm{~ms}$ & $1,25 \mathrm{~ms}$ & \\
\hline 임 Janela de modulação & Bartlett & Bartlett & \\
\hline$\sum_{0}^{0}$ Frequência do Tone Burst & $500 \mathrm{kHz}$ & $500 \mathrm{kHz}$ & $50 \mathrm{~Hz}$ \\
\hline Fase inicial do Tone Burst & 0 graus & 0 graus & \\
\hline ஸ Ciclos do Tone Burst & 1 & 1 & \\
\hline $\begin{array}{l}\text { Tipo do Levantamento } \\
\text { Sísmico }\end{array}$ & $\begin{array}{l}\text { Constant } \\
\text { Offset }\end{array}$ & $\begin{array}{l}\text { Constant } \\
\text { Offset }\end{array}$ & $\begin{array}{l}\text { End- } \\
\text { On }\end{array}$ \\
\hline $\begin{array}{l}\text { Espaçamento entre tiros da } \\
\text { : } \\
\text { : Fonte }\end{array}$ & $2 \mathrm{~mm}$ & $2 \mathrm{~mm}$ & $2 \mathrm{~m}$ \\
\hline 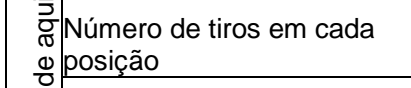 & 200 & 15 & \\
\hline $\begin{array}{l}\text { Números de tiros em cada } \\
\text { linha }\end{array}$ & 150 & 150 & \\
\hline \begin{tabular}{c|c}
$\bar{d}$ & Número total de tiros \\
$\circlearrowleft$
\end{tabular} & 30.000 & 2.250 & \\
\hline Número de linhas & 1 & 1 & \\
\hline Offset inicial & $40 \mathrm{~mm}$ & $34 \mathrm{~mm}$ & $340 \mathrm{~m}$ \\
\hline Lâmina d'água total no tanque & $200 \mathrm{~mm}$ & $200 \mathrm{~mm}$ & $2 \mathrm{~km}$ \\
\hline Número total de traços gerados & 150 & 150 & \\
\hline $\begin{array}{l}\text { Veloc. da onda } \mathrm{P} \text { (salmoura e } \\
\text { água, respectivamente) }\end{array}$ & $1540 \mathrm{~m} / \mathrm{s}$ & $1490 \mathrm{~m} / \mathrm{s}$ & \\
\hline $\begin{array}{l}\text { Velocidade da onda P no } \\
\text { Plexiglass }\end{array}$ & $2777 \mathrm{~m} / \mathrm{s}$ & $2777 \mathrm{~m} / \mathrm{s}$ & \\
\hline $\begin{array}{l}\text { Tempo de duração de uma } \\
\text { aquisição em laboratório }\end{array}$ & $6 \mathrm{~h}$ & $1 \mathrm{~h}$ & \\
\hline
\end{tabular}


A Figura 9 (superior e inferior) permite uma comparação entre os resultados obtidos por Silva, (2013) e os obtidos após a implementação das novas funcionalidades no sistema multicanal, durante a execução desta pesquisa. Os parâmetros usados nas 02 aquisições sísmicas estão descritos na Tabela 1.
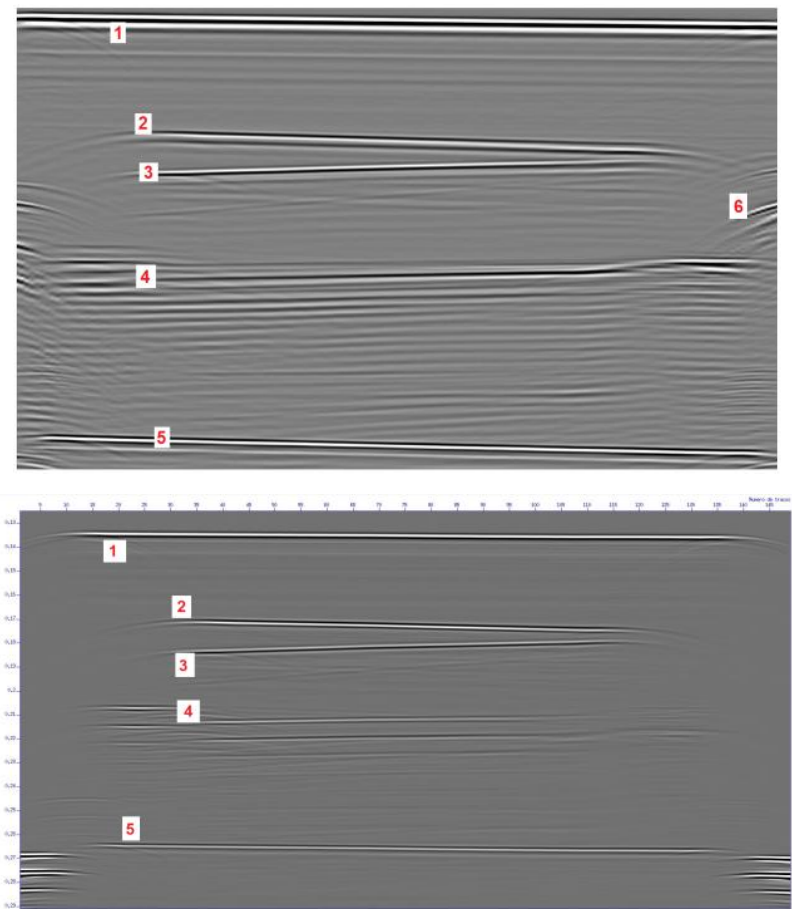

Figura 9 - (Superior) Imagem obtida com o sistema monocanal (Silva, 2013); (inferior) imagem obtida com o sistema multicanal. Eventos observados: 1. Topo do modelo. 2. Topo do reservatório. 3. Base do reservatório. 4. Base do modelo. 5. Múltipla. 6. Difração do efeito de borda.
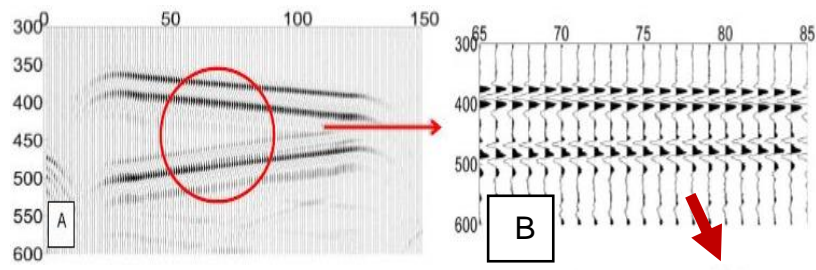

Figura 10: Sismograma expandido (Silva, 2013). (A) região do modelo físico compreendida entre os traços 1 - 150 e intervalo do reservatório entre 300 - 600, saturado por salmoura; $(B)$ região do reservatório entre os traços 65 - 85; (C) Detalhe ampliado do traço 75 . Observe a diferença entre as amplitudes das interfaces topo e base do reservatório, devido ao efeito de atenuação da onda $P$ e limitação do sistema monocanal.

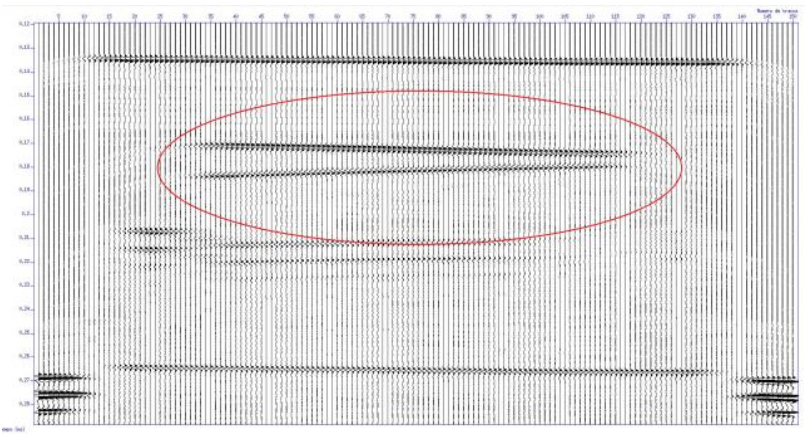

Figura 11 - Sismograma com um reservatório tipo cunha em destaque. Aquisição pelo sistema multicanal.

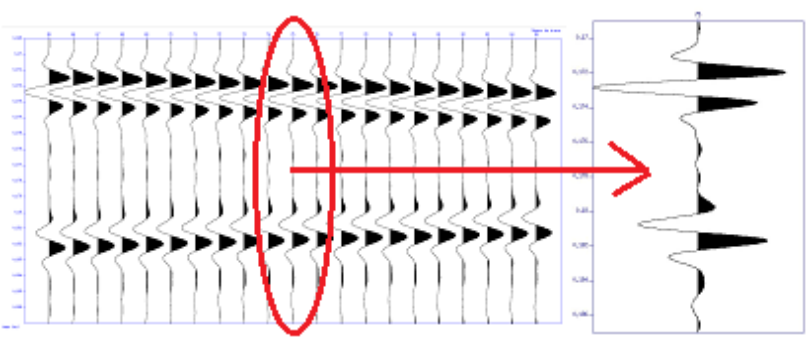

Figura 12 - (Esquerda) Sismograma ampliado na região entre traços 65 - 85 do reservatório preenchido com água; (Direita) o traço sísmico 75 ampliado.

\section{Discussão e Conclusões}

O protótipo do Sistema de Modelagem Física Sísmica Multicanal está projetado em sua totalidade para ser construído e implementado. As modificações realizadas no atual Sistema de Modelagem causaram impacto positivo, que podem ser vistos nos resultados dos experimentos para aquisição de dados sísmicos, Figuras 11 e 12. Por comparação das Figuras 10 (B e C) e 12 é possível visualizar a diferença entre as amplitudes das interfaces topo e base do reservatório. Este incremento de energia deve ser atribuído as novas funcionalidades (hardwere e softwere) inseridas ao sistema de modelagem que 0 habilitou a registros de dado multicanal, com maior conteúdo de energia.

Além disso, o software de desenvolvimento na plataforma LABVIEW está preparado para receber dados de até 32 canais com transdutores ultrassônicos. Durante a implementação do sistema multicanal houve a alteração na rotina de execução das geometrias de aquisição para evitar a inclusão de ruídos dos motores aos dados. Assim, foi inserido na programação do software, a parada total dos motores durante a aquisição dos dados sísmicos.

Outra melhoria significativa obtida em decorrência da implementação do Sistema de Modelagem Física Multicanal pode ser notada pelo pela redução do tempo total de duração do levantamento sísmico em laboratório. Conforme Tabelas 1, o levantamento sísmico que durava aproximadamente 6 horas, pode ser reduzido a cerca de 01 hora. 


\section{Agradecimentos}

Os autores agradecem ao LENEP/UENF pelas funcionalidades para este trabalho. SDRA agradece a PRH-PETROBRAS-226 pela bolsa de estudos e aos orientadores do doutorado por todo apoio e suporte técnico.

\section{Referências}

Assis, C.A.M., Ceia, M.A.R., Misságia, R.M., Lupinacci, W.M. (2014). Mapping fluid distribution in a pinch-out reservoir model: A physical Modeling study. Journal of Applied Geophysics.

Cordsen, Andreas; Galbraith, Mike; Peirce, John (2000). Planning land 3-D seismic surveys. Society of exploration geophysicists.

Ebrom, Daniel A.; McDonald, John Andrew (1994). Seismic physical modeling. Society of Exploration Geophysicists.

Kearey, Philip; Brooks, Michael; HILL, Ian. (2009) Geofísica de exploração. Oficina de Textos.

Misságia, R. M., Ceia, M., \& Pessanha, C. (2010). A Modelagem Física Sísmica na UENF/LENEP: descrição e teste do sistema. In IV Simpósio Brasileiro de Geofísica.

Panametrics. (2009). Manual of Ultra-sonic Transducers for Non-Destructive Tests. Fonte: http://www.olympusndt. com/en/panametrics-ndt-ultrasonic/.

Silva, J. P. (2013). Modelagem física dos efeitos da geometria do grão na resposta sísmica de reservatórios delgados com borda em forma de cunha (pinch-out). Dissertação de Mestrado. Universidade Estadual do Norte Fluminense.

Zambrini, J. P. R., (2015). Implementação de fluxo de processamento de dados sísmicos ultrassônicos com ênfase na migração e análise do atributo de atenuação sísmica. Dissertação de Mestrado. Universidade Estadual do Norte Fluminense. 